\title{
Mathematical Modeling for Corrosion Environment Estimation Based on Concrete Resistivity Measurement Directly above Reinforcement
}

\author{
Young-Chul LIM, ${ }^{11}$ Takafumi NOGUCHII) and Han-Seung LEE ${ }^{11}$ \\ 1) Sustainable Building Research Center, Hanyang University, Ansan, 426-791, Korea. E-mail: dpgyc919@gmail.com \\ 2) Department of Architecture, Faculty of Engineering, The University of Tokyo, Tokyo 113-8656 Japan.
}

(Received on July 16, 2008; accepted on October 10, 2008)

\begin{abstract}
This study aims to formulate a resistivity model whereby the concrete resistivity expressing the environment of steel reinforcement can be directly estimated and evaluated based on measurement immediately above reinforcement as a method of evaluating corrosion deterioration in reinforced concrete structures. It also aims to provide a theoretical ground for the feasibility of durability evaluation by electric non-destructive techniques with no need for chipping of cover concrete. This Resistivity Estimation Model (REM), which is a mathematical model using the mirror method, combines conventional four-electrode measurement of resistivity with geometric parameters including cover depth, bar diameter, and electrode intervals. This model was verified by estimation using this model at areas directly above reinforcement and resistivity measurement at areas unaffected by reinforcement in regard to the assessment of the concrete resistivity. Both results strongly correlated, proving the validity of this model. It is expected to be applicable to laboratory study and field diagnosis regarding reinforcement corrosion.
\end{abstract}

KEY WORDS: corrosion; nondestructive method; concrete; reinforcing bar; resistivity; mathematical model.

\section{Introduction}

Reinforcing steel in concrete, which is alkaline, is passivated by thin oxide film and protected from external corrosive action. However, environmental changes resulting from chloride attack due to chloride ion penetration and carbonation due to carbon dioxide diffusion facilitate the reaction of reinforcing steel with pore water and dissolved oxygen in concrete, activating corrosion deterioration. The growth of the resulting corrosion products causes cracking on concrete surfaces, ultimately impairing the reliability of concrete structures in terms of stability.

The methods of diagnosing corrosion deterioration are roughly classified into three groups: visual judgment of cracking and rust exudation, physical techniques including hammering, and electrochemical techniques ${ }^{1-4)}$ using halfcell potential, polarization resistance, A.C. impedance, etc. These techniques are selected and applied to each site depending on their characteristics, such as simplicity of diagnosis, quickness, reliability, and economical efficiency. However, problems remain unsolved in these techniques in regard to preventive diagnosis and concrete retention, such as judgment after significant deterioration and cover concrete chipping.

Several numerical models including finite element, ${ }^{5)}$ finite difference, ${ }^{6-8)}$ and boundary element method ${ }^{9-11)}$ have been developed to simulate corrosion of reinforcing materials embedded in concrete. Among them, a corrosion evalu- ation method using an inverse analysis method of the boundary element method has been conducted to identify the conductivity of concrete and the impedance at the boundary between reinforcement and concrete. ${ }^{12)}$ However, there are some problems in improving variations in the precision of the identification according to analysis conditions and measurement points.

The rate of the steel corrosion process in concrete structures is controlled by three mechanisms: anodic control, cathodic control, and resistance control. ${ }^{13)}$ Among these mechanisms, resistance control by the void percentage and moisture content of concrete predominantly governs the corrosion rate of reinforcing steel when the regions of anodic and cathodic corrosion are distant from each other. Therefore, Grasping the concrete resistivity is equivalent to knowing the state of electrolytes involved in corrosion reaction and obtaining information that enables indirect judgment of the corrosion environment surrounding reinforcement.

Resistivity measurement has been applied to concrete structures by arranging two each of current and potential electrodes on the concrete surface according to the Wenner method to detect the resistivity of concrete, ${ }^{14)}$ which is one of its physical properties. Measurement by this method has conventionally been carried out at unreinforced areas away from the object reinforcement to minimize its effect. However, indirect evaluation of a specific area based on the measurement at a distant area may blur the accuracy of 
judgment when the goal of diagnosis using concrete resistivity is to know the corrosion environment where there is a potential risk of corrosion deterioration.

On the other hand, a mathematical model using a Poisson equation in cylindrical coordinates was proposed by presenting a nondestructive method ${ }^{15-17)}$ that represents no damages on the cover of concrete in order to develop a measurement method that can be easily applied to the field. However, this study did not perform an experimental verification for the applicability of the proposed model even though this study presented a sense of direction for a complete nondestructive method.

With this as a background, the authors aim to propose a resistivity model that allows the estimation of the resistivity of concrete and reinforcement from the apparent resistivity measurement immediately above reinforcement in order to make an effective diagnosis on-site. In other words, this mathematical model is formulated on the basis of the resistivity of concrete and reinforcing bar, while previous mathematical models for corrosion estimation have ever been focused on the studies to represent the electrochemical response of corrosion.

The formation of this mathematical model using the mirror method is concerned with the concrete cover depth for the reinforcement, diameter of reinforcing bars, and electrode intervals as geometrical factors with the resistivity of concrete and reinforcement. Also, this study investigates the validity of the proposed model through the relationship between the results of the experimental measurement of the concrete resistivity and the calculation values estimated by this model.

\section{Resistivity Estimation Model}

When there is a point current source $(I)$ in an infinite isotropic homogeneous medium with a resistivity of $\rho$, the potential $(\phi)$ at a distance of $r$ from the point current source is written as Eq. (1).

$$
\phi=\frac{\rho \cdot I}{4 \pi r}
$$

Also, the potential $(\phi)$ generated by a point current source (I) on the surface of an infinite isotropic homogeneous medium with a resistivity of $\rho$ can be expressed as Eq. (2).

$$
\phi=\frac{\rho \cdot I}{2 \pi r}
$$

Resistivity measurement on a concrete surface has been carried out mostly by the Wenner method in which two outer current electrodes and two inner potential electrodes are arranged in line at equal intervals $(a)$. The apparent resistivity by this technique $(\rho)$ is derived as given in Eq. (3) in consideration of the potential difference $(V)$ generated between potential electrodes for the impressed current $(I)$ and the geometric factors of electrode configuration,

$$
\rho=\frac{V}{I} \cdot 2 \pi r
$$

where coefficient $a$ is the geometrical coefficient for the Wenner electrode configuration among the methods of fourelectrode resistivity measurement.
In actual field evaluation of concrete resistivity, judgment is prone to errors when it is based on the assumption that concrete is a semi-infinite isotropic homogeneous medium despite the inevitable inclusion of the effect of nearby reinforcement. It is therefore crucial for enhancing the accuracy of judgment to estimate the resistivity of concrete surrounding reinforcement by measuring directly above the reinforcement.

\subsection{Homogenization of Reinforcement Resistivity}

In order to simplify the resistivity estimation model, it is necessary to treat a reinforcing bar consisting of the internal conductor part and the external mill scale having different resistivities as a homogeneous single body. The resistivity of the entire cross-section of the cylindrical model is therefore assumed to be homogeneous.

\subsection{Resistivity Reflection Coefficient}

The present resistivity model expresses the potential distribution in two media having different resistivities by the mirror method. In order to use the mirror method for formulating the model, it is necessary to determine the resistivity reflection coefficient, ${ }^{18)}$ which is the size ratio of the current source to the mirror current source. In other words, the ratio of the point current source in medium $1(I)$ having a resistivity of $\rho_{1}$ to the mirror current source generated in medium 2 having a resistivity of $\rho_{2}$ has to be determined.

To begin with, reinforcement located within concrete with a resistivity of $\rho_{1}$ should be modeled as a cylinder with a resistivity of $\rho_{2}$, and the relative position of the mirror current source generated in the same cross-section by the current source in the outer system should be specified. As shown in Fig. 1, the positional relation of the current source $I$ and the mirror current source $I^{\prime}$ generated on the circular cross-section with respect to the origin as the datum point is determined as proportionality coefficient $k$ in Eq. (5) by the Apollonian circle. In a circle as shown in Fig. 1, the proportionality coefficient, $k$, expresses the relationship between point $I$ out of the circle and point $I^{\prime}$ on line $O I$ and within the circle to be $d: r=r: c$, where $d$ is the distance from the origin $(O)$ to $I, r$ is the radius, and $c$ is the distance from $O$ to $I^{\prime}$. This circle, which is the locus of point $P$ having this relationship, is an Apollonian circle.

$$
\begin{array}{r}
\frac{r}{d}=\frac{c}{r}=k \\
c=k r \ldots \ldots
\end{array}
$$

The case where medium 1 with a resistivity of $\rho_{1}$ is in contact with medium 2 with a resistivity of $\rho_{2}$ at the boundary of the cylinder $(S)$ as shown in Fig. 2 is then considered. When assuming the potential in medium 1 generated

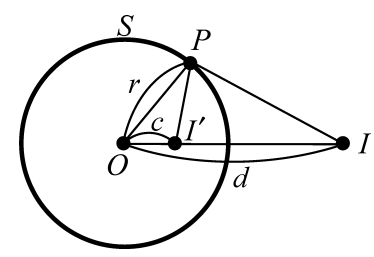

Fig. 1. Apollonian circle formed by the positional relationship between $I^{\prime}$ and $I^{\prime \prime}$. 




Fig. 2. Cylindrical coordinates for resistivity reflection coefficient.

by point current source $I$ in medium 1 and the potential in medium 2 to be $V_{1}$ and $V_{2}$, respectively, the boundary condition to be satisfied is the continuity of potential and current as given in Eqs. (6) and (7).

$$
\begin{array}{r}
V_{1}=V_{2} \ldots \ldots \ldots \\
\frac{1}{\rho_{1}} \frac{\partial V_{1}}{\partial z}=\frac{1}{\rho_{2}} \frac{\partial V_{2}}{\partial z}
\end{array}
$$

At point $P_{1}$ in medium 1 shown in Fig. 2, a potential of $V_{1}$ is generated by point current source $I$ and mirror point current source $I^{\prime}$ as given in Eq. (8). Also, potential $V_{2}$ generated at point $P_{2}$ in medium 2 by point current source $I$ and mirror point current source $I^{\prime}$ is determined from mirror current source $I^{\prime \prime}$ assumed again in medium 1 by $I^{\prime}$ and $I$ as given in Eq. (9) to eliminate an extreme value in medium 2.

$$
\begin{array}{ll}
V_{1}=\frac{\rho_{1}}{4 \pi}\left(\frac{I}{r_{1}}+\frac{I^{\prime}}{r_{1}^{\prime}}\right), \quad r \geq s \\
V_{2}=\frac{\rho_{1}}{4 \pi}\left(\frac{I}{r_{2}}+\frac{I^{\prime \prime}}{r_{2}^{\prime \prime}}\right), \quad r \leq s
\end{array}
$$

where $r=$ distance from $O$ to the local point: $r_{\mathrm{p} 1}, r_{\mathrm{p} 2}$ $s=$ radius

It should be noted that

$$
\begin{aligned}
r_{1}=r_{2} & =r_{2}^{\prime \prime}=\sqrt{(z-d)^{2}+x^{2}} \\
r_{1}^{\prime} & =\sqrt{(z-k s)^{2}+x^{2}} \ldots \ldots
\end{aligned}
$$

where

$$
\begin{aligned}
& z=r \cos \theta . \\
& x=r \sin \theta .
\end{aligned}
$$

By substituting these into the boundary conditions of Eqs. (6) and (7), the sizes of mirror current sources in both media are determined.

$$
\begin{gathered}
I^{\prime}=k I^{\prime \prime} \ldots \ldots \ldots \\
I^{\prime}=\left(\frac{k\left(\rho_{2}-\rho_{1}\right)}{\sqrt{k} \rho_{2}+\rho_{1}}\right) I
\end{gathered}
$$

The reflection coefficient $\left(Q_{1}\right)$ between point current

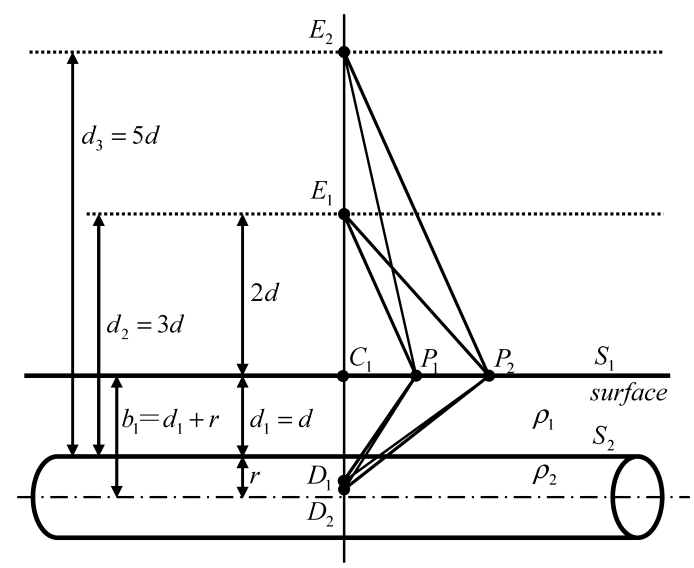

Fig. 3. Resistivity estimation model using the mirror method.

source $I$ and mirror current source $I^{\prime}$ and the reflection coefficient $\left(Q_{2}\right)$ between mirror current source $I^{\prime}$ and mirror current source $I^{\prime \prime}$ are written as follows:

$$
\begin{gathered}
Q_{1}=\left(\frac{k\left(\rho_{2}-\rho_{1}\right)}{\sqrt{k} \rho_{2}+\rho_{1}}\right) . \\
Q_{2}=k \ldots \ldots \ldots \ldots \ldots
\end{gathered}
$$

\subsection{Formulation of Resistivity Estimation Model}

This study aims to formulate a resistivity model whereby the concrete resistivity can be estimated from the apparent resistivity of concrete surrounding the reinforcement to be diagnosed by measuring directly above the reinforcement to enhance the accuracy of corrosion evaluation. This model employs the Wenner method, in which two outer current electrodes and two inner potential electrodes are arranged in line at equal intervals $(a)$, from among resistivity measuring methods using four electrodes. Changes in the potential distribution involving different resistivities such as concrete and reinforcement can be expressed by the mirror method. ${ }^{18,19)}$

Figure 3 shows the relations between potentials at points $P_{1}$ and $P_{2}$ on the same line generated by current source $I_{C_{1}}$ placed at point $O$ on the measurement line on the concrete surface parallel to the reinforcement. The total space consisting of three elements, the air layer $\left(\rho_{\text {air }}=\infty\right)$, concrete $\left(\rho_{\text {concrete }}=\rho_{1}\right)$, and reinforcement $\left(\rho_{\text {bar }}=\rho_{2}\right)$, is regarded as a homogeneous medium with a resistivity of $\rho_{1}$, and current $I_{C_{1}}(2 I)$ is placed at the origin $(O)$ on the concrete surface. The potential $\left(V_{C_{1}}\right)$ generated by this at point $P_{1}$ is written as Eq. (18).

$$
V_{C_{1}}=\frac{\rho_{1} I}{2 \pi} \frac{1}{a}
$$

A mirror current source for current source $I_{C_{1}}$ with respect to boundary $S_{2}$ is then placed at position $D_{1}$. The potential $\left(V_{D_{1}}\right)$ generated by mirror current source $I_{D_{1}}$ at point $P_{1}$ is written as Eq. (19).

$$
V_{D_{1}}=\frac{\rho_{1} I}{2 \pi}\left[\frac{Q_{1}}{\left\{a^{2}+\left(d+r\left(1-k_{1}\right)\right)^{2}\right\}^{1 / 2}}\right] \ldots
$$


If mirror current source $I_{D_{1}}$ is placed, then mirror current source $I_{E_{1}}$ is necessary to satisfy the boundary condition of boundary $S_{1}$. If mirror current source $I_{E_{1}}$ is placed, then mirror current source $I_{D_{2}}$ is necessary to satisfy the boundary condition of boundary $S_{2}$. The potential at point $P_{1}$ is thus determined one after another as follows:

$$
\begin{gathered}
V_{E_{1}}=\frac{\rho_{1} I}{2 \pi}\left[\frac{1}{k_{1}} \frac{Q_{1}}{\left\{a^{2}+(2 d)^{2}\right\}^{1 / 2}}\right] \ldots \ldots \ldots \ldots . . . . \\
V_{D_{n}}=\frac{\rho_{1} I}{2 \pi}\left[\left[\prod_{n=1}^{m} \frac{Q_{n}}{k_{n}}\right] \cdot\left[\frac{k_{n}}{\left\{a^{2}+\left(d+r\left(1-k_{n}\right)\right)^{2}\right\}^{1 / 2}}\right]\right] \ldots \\
V_{E_{n}}=\frac{\rho_{1} I}{2 \pi}\left[\left[\prod_{n=1}^{m} \frac{Q_{n}}{k_{n}}\right] \cdot\left[\frac{k_{n}}{\left\{a^{2}+(2 n d)^{2}\right\}^{1 / 2}}\right]\right] \ldots \ldots
\end{gathered}
$$

Therefore, potential $V_{11}$ at point $P_{1}$ by point current source $I_{C_{1}}$ is determined as Eq. (23).

$$
\begin{aligned}
V_{11} & =V_{C_{1}}+V_{D_{1}}+V_{E_{1}}+\cdots+V_{D_{n}}+V_{E_{n}}+\cdots \\
& =V_{C_{1}}+\sum_{n=1}^{\infty}\left(V_{D_{n}}+V_{E_{n}}\right) \ldots \ldots \ldots \ldots \ldots \ldots \ldots \ldots \ldots \ldots \ldots \ldots \ldots \ldots
\end{aligned}
$$

The potential difference $\left(V_{\mathrm{a}}\right)$ between $P_{1}$ and $P_{2}$ in the electrode configuration in measurement by the Wenner method is expressed as Eq. (24) following the potential combining equation $V_{\mathrm{a}}=\left(V_{11}+V_{21}\right)-\left(V_{12}+V_{22}\right)$ by assuming the potentials at $P_{1}$ and $P_{2}$ by $I_{C_{1}}(I)$ to be $V_{11}$ and $V_{12}$ and by $I_{C_{2}}(-I)$ to be $V_{21}$ and $V_{22}$.

$$
\begin{aligned}
V_{\mathrm{a}}= & \frac{\rho_{1} \cdot I}{\pi a}\left[\frac{1}{2}+\sum_{n=1}^{\infty}\left[\left[k_{n} \prod_{n=1}^{\infty} \frac{Q_{n}}{k_{n}}\right]\right.\right. \\
& \cdot\left[\frac{1}{\left(1+H_{n}^{2}\right)^{1 / 2}}-\frac{1}{\left(4+H_{n}^{2}\right)^{1 / 2}}\right] \\
& \left.+\left[\prod_{n=1}^{\infty} \frac{Q_{n}}{k_{n}}\right] \cdot\left[\frac{1}{\left(1+G_{n}^{2}\right)^{1 / 2}}-\frac{1}{\left(4+G_{n}^{2}\right)^{1 / 2}}\right]\right] \ldots
\end{aligned}
$$

It should be noted that:

$$
\begin{gathered}
k_{n}=\frac{r}{(1+2(n-1)) d+r} \\
Q_{n}=\frac{k_{n}\left(\rho_{2}-\rho_{1}\right)}{\sqrt{k_{n}} \rho_{2}+\rho_{1}} \ldots \\
H_{n}=\frac{d+r\left(1-k_{n}\right)}{a} \ldots \\
G_{n}=\frac{2 n d}{a} \ldots \ldots \ldots . . .
\end{gathered}
$$

where $V_{\mathrm{a}}=$ apparent potential difference $(\mathrm{V})$

$\rho_{1}=$ concrete resistivity $(\Omega \cdot \mathrm{m})$

$\rho_{2}=$ reinforcement resistivity $(\Omega \cdot \mathrm{m})$

$d=$ concrete cover depth $(\mathrm{m})$

$r=$ reinforcement radius $(\mathrm{m})$

$a=$ interval between electrodes (m)
This is the Resistivity Estimation Model (REM) intended in this study for estimating the resistivity when a reinforcing bar is present directly under the concrete. Resistivity values can be estimated from the measurements by error minimization.

\subsection{Effects of Geometrical Factors}

In order to grasp the effects of geometrical factors included during the measurement of the apparent resistivity, an analytical examination was made to this REM. Figure 4 shows the changes in terms of response voltage $(V)$ by changing the electrode intervals $(a=0.02,0.03$, and $0.04 \mathrm{~m})$, concrete cover depth $(0.01$ to $0.1 \mathrm{~m})$, and reinforcement diameter $(0.01$ to $0.05 \mathrm{~m})$ while keeping the concrete resistivity $\left(\rho_{1}\right)$ and reinforcement resistivity $\left(\rho_{2}\right)$ constant at $100 \Omega \cdot \mathrm{m}$ and $0 \Omega \cdot \mathrm{m}$, respectively. The homogeneous state without reinforcement is also included in the figure to examine the effect of reinforcement.

As for the effect of electrode intervals, the response voltage $(V)$ decreases as the interval increases, approaching $0 \mathrm{~V}$ as the interval approaches infinity as given in Eq. (3). The response voltage rapidly increases as the cover depth increases in the beginning but gradually levels off, converging with the response voltage for homogeneous concrete without reinforcement. This is related to the current path at the time of measurement, i.e., the volumetric ratio of reinforcement to concrete within the range of measurement, expressing that the current flows in only concrete as the distance between the measuring point and reinforcement increases. Though the response voltage $(V)$ decreases as the bar diameter increases, the differences are smaller than in the case of cover depth, presumably because the volumetric change within the current-carrying range is smaller than in the case of cover depth.

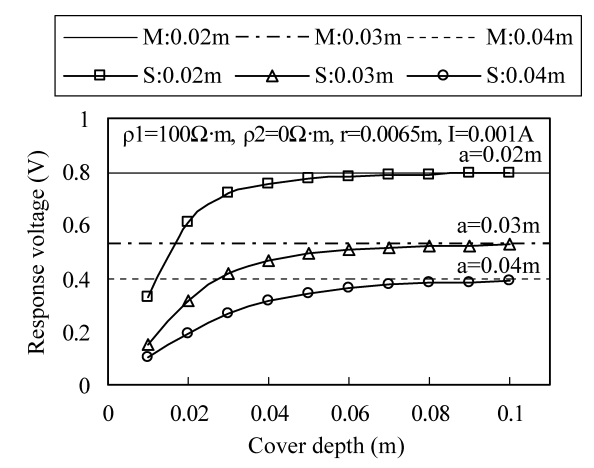

(a) Effect of cover depth

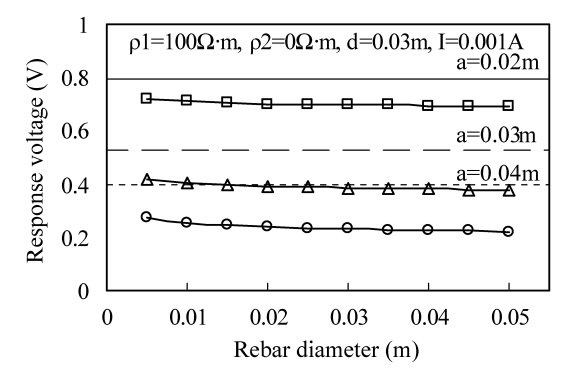

(b) Effect of Rebar diameter

Fig. 4. Simulation of response voltage related to geometric parameters. 


\section{Outline of Experiment}

The apparent resistivity measurements of concrete are prone to scatter even within the same mixture or specimen depending on the material composition, void percentage, and moisture content. Adequate evaluation of concrete resistivity can therefore provide important information for judgment regarding the deterioration prediction of structures.

The validity of the model for estimating concrete resistivity was experimentally verified, using specimens having different cover depths and bar diameters, by measuring the resistivity of concrete at points judged as being unaffected by reinforcement and estimating the concrete resistivity using the resistivity model based on measurement directly above reinforcement.

\subsection{Specimen Fabrication}

As shown in Fig. 5, mortar specimens measuring 400 by 400 by $100 \mathrm{~mm}$ were fabricated using normal portland cement with a water-cement ratio (W/C) of $60 \%$ and cement-sand ratio of $1: 2$. Reinforcing bars were cut to $41 \mathrm{~cm}$ and immersed in a $10 \%$ aqueous solution of diammonium citrate for $2 \mathrm{~d}$ to remove mill scale. The end $35 \mathrm{~mm}$ on both sides of each bar was coated with epoxy to eliminate the direct effects of external factors.

The reinforcement surfaces to be exposed to mortar was washed with acetone and immediately fixed to molds in one direction to be made into specimens with the specified levels of factors, as shown in Table 1. Specimens were demolded $2 \mathrm{~d}$ after placing and subjected to water curing at $20^{\circ} \mathrm{C}$ for $45 \mathrm{~d}$ until measurement.

\subsection{Measuring Device}

The resistivity measurement is normally carried out by applying a sinusoidal current with a frequency ranging from 50 to $1000 \mathrm{~Hz}^{4)}$ to current electrodes placed on the specimen surface according to the Wenner method and measuring the potential difference between the voltage electrodes generated by the forced polarization. In the present test, a frequency of $100 \mathrm{~Hz}$ was selected for measurement based on preliminary test results. A function generator manufactured by Yokogawa Electric Corporation (PC base WE 7000) was used to stabilize the measuring operation. Figure 6 shows the measurement setup.

\subsection{Test Procedure}

In order to estimate the concrete resistivity expressing the corrosion environment of reinforcement by measuring the apparent resistivity directly above the specified reinforcing bars, tests began with identifying an area for measuring the apparent resistivity on the same specimen unaffected by nearby reinforcement, so as to compare the measurement with the estimated value.

The sides of each specimen were coated with epoxy to prevent moisture evaporation from concrete during measuring and keep the resistivity condition of concrete in a homogeneous manner. In other words, specimens removed from water were left to stand in air at $20^{\circ} \mathrm{C}$ and $55 \%$ R.H. for $12 \mathrm{~h}$ to dry their surfaces. The four sides of specimens were then coated with epoxy, while leaving the top and bot-

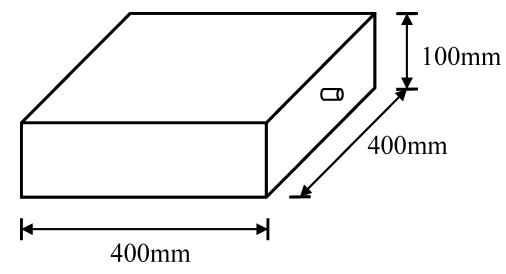

Fig. 5. Geometry of mortar specimens.

Table 1. Geometric factors of specimens.

\begin{tabular}{c|c|c}
\hline Specimen & Cover depth $(\mathrm{mm})$ & Rebar diameter $(\mathrm{mm})$ \\
\hline $\mathrm{T} 20-\varphi 13$ & 20 & 13 \\
\hline $\mathrm{T} 30-\varphi 13$ & 30 & 13 \\
\hline $\mathrm{T} 40-\varphi 13$ & 40 & 13 \\
\hline $\mathrm{T} 30-\varphi 19$ & 30 & 19 \\
\hline $\mathrm{T} 30-\varphi 25$ & 30 & 25 \\
\hline
\end{tabular}

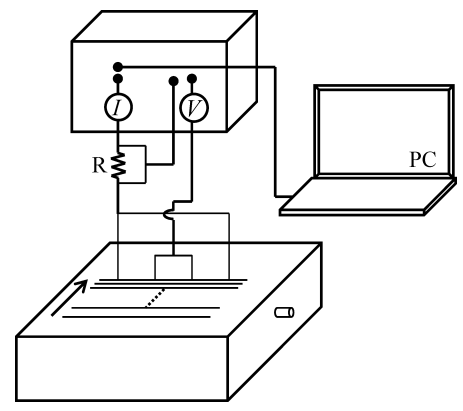

Fig. 6. Schematic diagram of measurement setup.

tom surfaces uncoated to ensure moisture transfer during measurement and re-curing. After drying the epoxy, these were water-cured again for $2 \mathrm{~d}$.

Specimens were removed from water immediately before measurement, with the water on the surfaces being wiped off, and marked to indicate the measuring points. A conductive gel with a resistivity of $8.3 \times 10^{-6} \Omega \cdot \mathrm{m}$ was applied in circular forms $3 \mathrm{~mm}$ in diameter to the four positions of electrodes on each specimen, and copper wire $1 \mathrm{~mm}$ in diameter was fixed to the gel spots, avoiding moving, to form an electric circuit together with the measuring device.

The applied current was a $1 \mathrm{~mA}$ sinusoidal current with a frequency of $100 \mathrm{~Hz}$, which can be regarded as short circuit for capacitance, in consideration of not only concrete but also the effect of electric double layer at the boundary between concrete based on the findings obtained from preliminary testing.

As shown in Fig. 7, the electrodes were arranged in parallel with reinforcing bars according to the Wenner method, and the apparent resistivity was measured along a traverse line perpendicular to the reinforcing bar, beginning from a distance toward the line directly above the bar $(14,10,7,5$, $4,3,2,1$, and $0 \mathrm{~cm}$ from the bar), so as to define the influence range of reinforcement on the apparent resistivity.

\subsection{Method of Calculating Apparent Resistivity}

The apparent resistivity was calculated by using a geometric factor referred to as the electrode configuration coefficient $(K)$ to incorporate this. The electrode configuration 


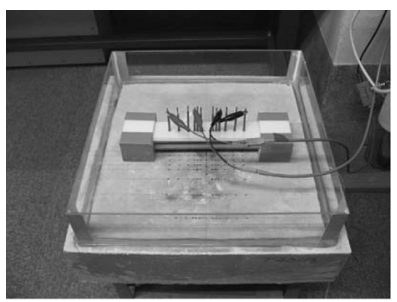

(a) Front view

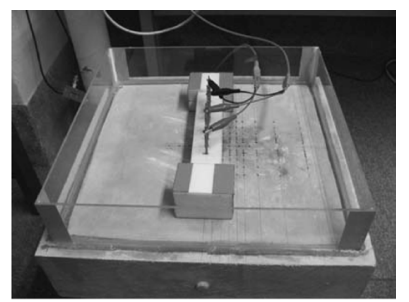

(b) Side view
Fig. 7. Photographs of a specimen with electrode setup.

coefficient for the Wenner configuration is $2 \pi a$.

$$
\rho=\frac{V}{I} \cdot K
$$

where $\rho(\omega)=$ apparent resistivity $(\Omega \cdot \mathrm{m})$

$I, V=$ amplitudes of input current and response voltage $(\mathrm{mA}, \mathrm{mV})$

$K=$ electrode configuration coefficient $(=2 \pi a$, Wenner method) $(\mathrm{m})$

$a=$ electrode interval (m)

\subsection{Test Results}

The results of tests on the effects of geometric factors e are summarized in Fig. 8.

\subsubsection{Effect of Cover Depth}

To examine the changes in the apparent resistivity with different cover depths $(d=2,3$, and $4 \mathrm{~cm})$, measurements were made on specimens with the same bar diameter $(13 \mathrm{~mm})$ by moving the measurement line along one side of the specified bar from a distance to directly above the center of the bar diameter, stopping at nine positions $(14,10,7$, $5,4,3,2,1$, and $0 \mathrm{~cm}$ from directly above the bar). The effect of the electrode intervals was also examined by changing the intervals $(a=2,3$, and $4 \mathrm{~cm})$ at each measurement position to carry out two-dimensional evaluation.

These tests revealed that the apparent resistivity decreased as the cover depth decreased with the same electrode intervals and as the electrode intervals increased with the same cover depth, showing a greater effect of reinforcement. ${ }^{20)}$ Since the changes in the apparent resistivity are particularly evident near the bar, cover depth may serve as a primary vertical parameter for reinforcement detection in the future. It should be noted, however, a greater cover depth and greater electrode intervals lead to significant increases in the effect of mortar compared with that of reinforcement within the measurement range, ultimately leveling off when these parameters exceed certain levels.

Though with a slight scatter among specimens, the apparent resistivity curves express the effect of cover depth as expected. It is therefore found that the judgment of concrete resistivity should naturally be based on measurement directly above reinforcement.

\subsubsection{Effect of Bar Diameter}

To examine the effect of the bar diameter, measurements were made on specimens with the same cover depth $(d=3 \mathrm{~cm})$ and different bar diameters $(\phi=13,19$, and $25 \mathrm{~mm}$ ) by moving the electrode line similarly to the test

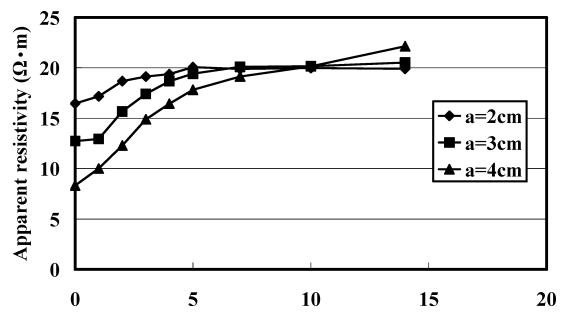

(a) $\mathrm{T} 20-\phi 13$

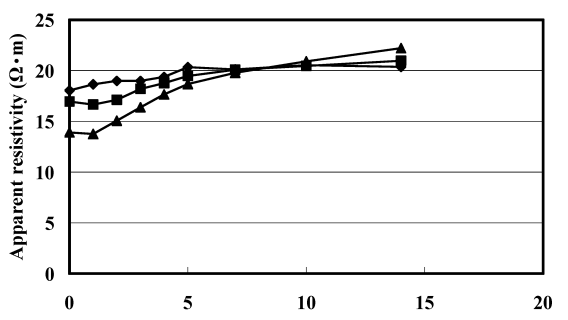

(b) $\mathrm{T} 30-\phi 13$

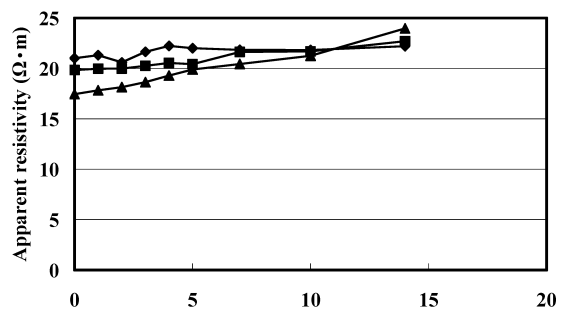

(c) $\mathrm{T} 40-\phi 13$

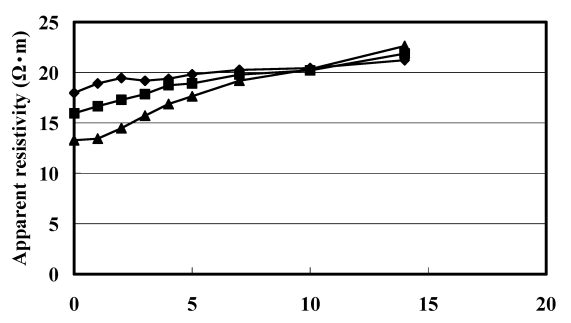

(d) $\mathrm{T} 30-\phi 19$

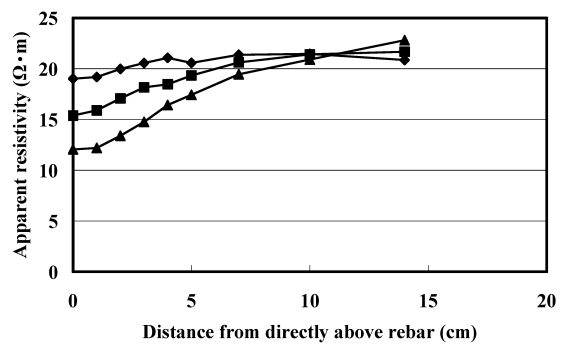

(e) T30- $\$ 25$

Fig. 8. Influence of changing cover depth and rebar diameter on apparent resistivity.

with different cover depths, while changing the electrode intervals $(a=2,3$, and $4 \mathrm{~cm})$. As a result, the apparent resistivity decreased as the bar diameter increased and as the electrode interval increased. However, the effect of bar diameter was much smaller than the effect of cover depth.

This is presumably because the current path is more strongly affected by the changes in the concrete volume due to changes in the cover depth or electrode intervals than by the changes in the bar diameter (bar volume). In other words, not only the resistivity of the media but also the vol- 
ume ratio of the media included in the measurement range is found to be a key factor for determining the apparent resistivity. Also, changes in the bar diameter of the circular cross-section are found to have a greater horizontal effect than a vertical effect on the apparent resistivity.

Though the values slightly scattered from one measurement position to another, the general tendencies are clearly defined. A distance of $10 \mathrm{~cm}$ from the reinforcing bar was selected as the area unaffected by reinforcement, i.e., the optimum area for measuring the apparent resistivity of mortar alone, as the scatter was smallest at this distance for each electrode interval. Increases in the apparent resistivity at a larger distance from the bar were attributed to the effect of the geometrical factor of the sides of specimens.

\section{Model Verification}

\subsection{Estimation of Concrete Resistivity}

This section discusses the validity of the proposed resistivity model in regard to the estimation of concrete resistivity around reinforcement based on measurement directly above the reinforcement. Model verification tests were carried out using five types of specimens with different cover depths and reinforcing bar diameters.

The resistivity of concrete and reinforcement were estimated by error minimization using the present model and measurements obtained directly above reinforcement with three different electrode intervals ( $a=2,3$, and $4 \mathrm{~cm}$ ). Also, the apparent resistivity of concrete alone was determined by averaging the measurement with three different electrode intervals $(a=2,3$, and $4 \mathrm{~cm})$ on the premise that it is expressed by measurement at around $10 \mathrm{~cm}$ from the reinforcement.

Figure 9 shows the relationship between the concrete resistivity values estimated by the proposed resistivity model and the measured values. Both are strongly correlated as if they all agree, though with a slight scatter due to specimens and positions of measurement, proving that the present resistivity model accurately estimates the concrete resistivity from measurement directly above reinforcement. The resistivity of reinforcement estimated simultaneously as concrete was mostly $1 \Omega \cdot \mathrm{m}$ or less, though with a certain scatter. These are not shown here, as this study primarily deal with the resistivity of concrete and the resistivity of reinforcement is assumed to be $0 \Omega \cdot \mathrm{m}$.

\subsection{Effects of Cover Depth and Bar Diameter}

Figures 10 show the measured voltage values regarding changes in the cover depth and bar diameter shown in Fig. 8 and voltage curves obtained by substituting the estimation by the resistivity model into the same geometrical conditions. Note that the resistivity of reinforcement $\left(\rho_{2}\right)$ is assumed to be $0 \Omega \cdot \mathrm{m}$.

This figure reveals that the resistivity model accurately expresses the changes due to these factors, particularly confirming the effect of cover depth being greater than that of bar diameter. Accordingly, the validity of this resistivity model is verified.

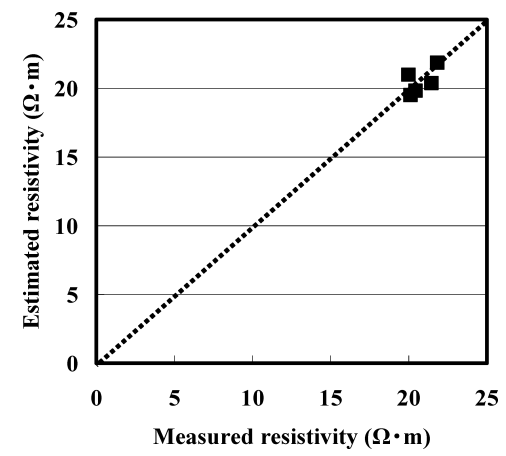

Fig. 9. Relationship between estimated and measured resistivities.

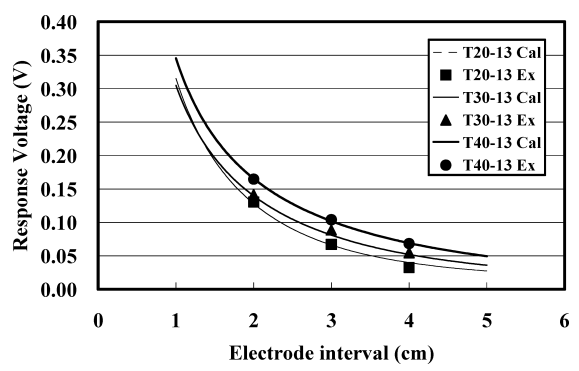

(a) Cover depth

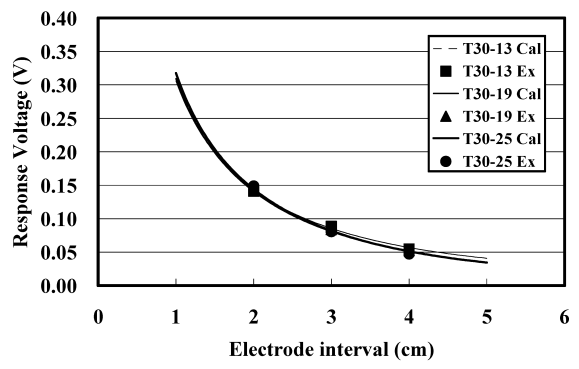

(b) Rebar diameter

Fig. 10. Relationship between calculated and measured voltage values and geometric parameters.

\section{Summary}

A resistivity model by a completely nondestructive technique was proposed in this study using the mirror method to estimate the concrete resistivity, which forms the corrosion environment of steel reinforcement in reinforced concrete structures. It enables grasping of changes in the potential within concrete by apparent resistivity measurement when a circumferential system, such as reinforcement, is present in a semi-infinite isotropic homogeneous medium.

The resistivity of specimens having different cover depths and bar diameters were measured and estimated to experimentally verify the validity of the model, and the following results and findings were obtained:

(1) The resistivity of reinforcement was assumed to be homogeneous to derive the resistivity reflection coefficient for a circular cross-section. Then the Resistivity Estimation Model (REM) was formulated using this coefficient and concerned with the concrete cover depth for the reinforcement, diameter of reinforcing bars, and electrode intervals as geometrical factors.

(2) In the evaluation of geometrical factors, the fact that the effect of cover depth was greater than that of rein- 
forcing bar diameter by measurement was also evident in the verification of the resistivity curves obtained using the model. This phenomenon is related to the path of the applied current. The changes in the cover depth, for instance, were found to change the volume of the current path more than the changes in the bar diameter do.

(3) The values estimated by this model directly above reinforcement were strongly correlated with the values measured at points unaffected by reinforcement. Resistivity estimation using this resistivity model was therefore judged as being valid. The application of this model to a system involving inhomegeneity and voids, such as concrete, should be investigated from now on.

(4) The applicability of this model should be widened in the future to include the estimation of changes in the bar resistivity as corrosion proceeds, so as to make this model more readily applicable to laboratory and field evaluation of corrosion environment.

The values estimated by this model directly above reinforcement were strongly correlated with the values measured at points unaffected by reinforcement. Resistivity estimation using this resistivity model was therefore judged as being valid. The application of this model to a system involving inhomegeneity and voids, such as concrete, should be investigated from now on.

\section{Acknowledgments}

This work on an early draft was advised to Professor Takafumi Noguchi as a supervisor when the first author on this paper studied it at the doctoral course in the University of Tokyo. It was also supported by Sustainable Building Research Center of Hanyang University which was supported the SRC/ERC program of MEST (Grant \# R112005-056-04003-0).

\section{REFERENCES}

1) I. Martinez, C. Andrade, N. Rebolledo, V. Bouteiller, E. Marie-Victoire and G. Olivier: Corrosion, 64 (2008), 107.

2) J. A. Gonzalez, J. M. Miranda N. Birbilis and S. Feliu: Corrosion, 61 (2005), 37.

3) J. A. Gonzalez, J. M. Miranda and S. Feliu: Corros. Sci., 46 (2004), 2467.

4) J. A. Gonzalez, A. Cobo, M. N. Gonzalez and S. Feliu: Corros. Sci., 43 (2001), 611

5) L. Bertolini, F. Bolzoni, L. Lazzari, T. Pastore and P. Pedeferri: Corros. Sci., 35 (1993), 1633.

6) F. Cui and A. A. Sagüés: Corrosion, 62 (2006), 918.

7) S. C. Kranc and A. A. Sagüés: Corrosion, 50 (1994), 50.

8) S. C. Kranc and A. A. Sagüés: Corros. Sci., 43 (2001), 1355.

9) M. Ridha, K. Amaya and S. Aoki: Corrosion, 57 (2001), 794.

10) M. Ridha, K. Amaya and S. Aoki: Corrosion, 61 (2005), 784.

11) Wessex Institute of Technology: Simulation of Elcetrochemical Processes II, WIT Press, Southampton, Boston, (2007), 299.

12) M. Yoshida, M. Ridha, K. Suga, K. Amaya and S. Aoki: The Japan Society of Mechanical Engineers, 12 (2006), 215.

13) Japan Society of Corrosion Engineering: Corrosion Engineering Handbook, Maruzen, Tokyo, (2000), 216.

14) R. Polder, C. Andrade, B. Elsener, $\Phi$. Vennesland, J. Gulikers, R. Weidert and M. Rqupach: Mater. Struct., 33 (2000), 603.

15) P. J. M. Monteriro, F. Morrison and W. Frangos: ACI Mater. J., 95 (1998), 704.

16) J. Zhang, P. J. M. Monteiro and H. F. Morrison: ACI Mater. J., 98 (2001), 116.

17) J. Zhang, P. J. M. Monteiro and H. F. Morrison: ACI Mater. J., 99 (2002), 242.

18) K. Sassa, T. Kanno and Y. Ashida: Geophysical Exploration for Construction and Disaster Prevention Engineers, Morikita Press, Tokyo, (1999), 148

19) The Society of Exploration Geophysicists: Exploration of Geophysicsts' Mining Geophysics, George Banta Co., Oklahoma, (1967), 63

20) J. Zhang, P. J. M. Monteiro, H. F. Morrison and M. Mancio: $A C I$ Mater. J., 101 (2004), 273. 